\title{
LOW FREQUENCY NOISE CONVERSION \\ IN FETS UNDER NONLINEAR OPERATION
}

\author{
F. DANNEVILLE, B. TAMEN, A. CAPPY \\ J-B JURAVER*, O. LLOPIS*, J. GRAFFEUIL* \\ IEMN, UMR CNRS 8520, Avenue Poincaré, BP 69, 59652 Villeneuve d'Ascq, France \\ *LAAS-CNRS, 7 Av. du Colonel Roche, 31077 Toulouse, France \\ francois.danneville@iemn.univ-lille1.fr
}

\begin{abstract}
Based upon the active line concept, the conversion mechanisms of microscopic low frequency noise (e.g. generation-recombination noise) located in the channel of a Field Effect Transistor (FET) which is driven by a large RF signal is demonstrated. The first consequence is that the based band (low frequency) input gate noise voltage spectral density is dependent on the magnitude of the input RF power applied to the FET. Moreover, the microscopic generation-recombination noise sources located in the channel are responsible of up-converted input gate noise voltage spectral density around the RF frequency.
\end{abstract}

\section{INTRODUCTION}

One of the most difficult problem encountered in FET noise modeling concerns the study of up-conversion of base-band low frequency $(L F)$ noise around a RF signal. Basically, part of the LF noise components is located at the surface between source and gate, or can be located in the substrate ; this leads generally to noise sources associated with extrinsic resistances such the gate resistance or source resistance. However, part of the noise can be also located in the channel of the FET ; this noise is related to carriers number fluctuation, and is called generation-recombination noise $(G R)$. The only way to model such a phenomena is to develop a physical noise modeling, close to the device physics ; one solution passes through the use of an active line to describe the FET, which is described in this paper.

\section{THEORETICAL ANALYSIS}

\section{(i) Large Signal Analysis}

In order to conduct the large signal analysis, let us consider the intrinsic part of the FET described by a uniform active line, and that the device operates as the active part of a FET amplifier, under large signal operation (Fig. 1). We assume that the large-signal steady state $V_{g s}(t)$ and $V_{d s}(t)$ resulting from the application of the input large $R F$ signal of angular frequency $\omega_{0}$ is known (determined using a harmonic balance simulator). The elements of the line, e.g. the local transconductance $g_{m}\left(V_{g s}, V_{d s}\right)$, the local channel conductance $\Delta g_{0}\left(V_{g s}, V_{d s}\right)$, and the local gate to channel capacitance $\Delta c_{0}\left(V_{g s}, V_{d s}\right)$ of the line, are related to experimental variations of the FET non-linear elements $G_{m}\left(V_{g s}, V_{d s}\right), G_{d}\left(V_{g s}, V_{d s}\right), C_{g s}\left(V_{g s}, V_{d s}\right)$, and $C_{g d}\left(V_{g s}, V_{d s}\right)$, which can be determined quite easily. Since time-varying voltages $V_{g s}(t)$ and $V_{d s}(t)$ are applied to the line, the line elements are also time dependent and can be developed in Fourier Series.

In the following, we are going to describe the different steps leading to the noise correlation matrix (NCM) calculation of the FET.

\section{(ii) Noise Analysis}

If we consider small perturbations around the large signal steady state, each element of the line can be replaced by its conversion matrix. The elements of the conversion matrix are determined in a straightforward way by the Fourier components of $g_{m}(t), \Delta g_{0}(t)$ and $\Delta c_{0}(t)$. To perform the noise analysis, we have chosen a representation with a open circuit between gate and source and a short circuit between drain and source (Fig. 2). A key point is to define the spectral density $S_{i n}$ of the local channel noise sources $\left\langle i_{n}{ }^{2}\right\rangle$ (Fig. 2), which is considered also as uniforms. A $G R$ microscopic noise source owns characteristics time $\tau_{c}$ ranged typically between $1 \mu s$ and $1 m s$. This means that the period of the microwave signal will be much lower than $\tau_{c}$, consequently, we will consider that $\left\langle i_{n}{ }^{2}\right\rangle$ is not modulated by the large signal. A problem also encountered is to relate $S_{i n}$ to the spectral density $S_{e}$ of the external low-frequency input gate noise voltage $\left\langle\mathrm{e}^{2}>\right.$ (Fig. 1), determined experimentally using a low frequency noise measurement system. The following relationship can 
be derived from a linear noise analysis of the circuit shown in Fig. 2, when the FET is biased in saturation (active operation) :

$$
S_{i n}=\left(\frac{L}{d x}\right) * g 0_{D C} * g m_{D C} * 2 * S e_{G R}
$$

$\mathrm{L}$ is the gate length, $S e_{G R}$ is the Generation-Recombination noise component of $S e, g O_{D C}$ and $g m_{D C}$ correspond respectively to the $\mathrm{DC}$ values of the transconductance and channel conductance. Once the microscopic noise sources are defined, the objective lies in the determination of the noise sources in the frequency domain at the input and output of the device, taking into account of the particular representation. The method is similar to those we have used previously in the case of FET mixer noise analysis [1]. By the end of the calculation, the FET can be described by its noise conversion and noise correlation matrices (Fig. 3 ). The input ports and output ports are defined for each angular frequency $\omega+K . \omega_{0}$, with $\omega_{0}$ corresponding to the RF angular frequency and $\omega$ to the angular frequency deviation from $\omega_{0}$. Note that the $1 / \mathrm{f}$ component of $\left\langle e^{2}\right\rangle$ (Fig. 1) is obviously added to the base band noise source $\left\langle e g_{0}^{2}\right\rangle$ of this circuit.

\section{RESULTS}

We have performed a complete simulation of a $F E T$ amplifier driven by a $R F$ signal at $3.5 \mathrm{GHz}$. The input and output RF terminations of the amplifier are simply $50 \Omega$, the device is a pseudomorphic HEMT $\left(0.25 * 200 \mu \mathrm{m}^{2}\right)$ biased at $V_{g s}=O \mathrm{~V}$ and $V_{d s}=2 \mathrm{~V}$. It was found that the correlation between any input gate noise voltage and any output drain noise current (Fig. 3) is zero, which is consistent with what has been observed in FET mixer noise analysis [1]. Moreover, due to the uniform character of the line, there is no conversion around the RF angular frequency for the drain noise current source; this not at all the case for the input gate noise voltage, which has strong consequences on the phase noise presented by FET oscillator [2]. For this purpose, we have performed simulations of the amplifier for a large variation of the input $R F$ power magnitude $P_{i n}$. The spectral densities of the theoretical and experimental base-band voltage noise source at the input $\left\langle e g_{0}{ }^{2}(\omega)\right\rangle$ (including flicker and $G R$ noise components) are represented on Fig.4.

We observe Fig.4b that the spectral density $\operatorname{Seg}_{0}(\omega)$ of the base-band voltage noise $\left\langle e g_{0}{ }^{2}(\omega)\right\rangle$ depends strongly on the magnitude of the input power magnitude $P_{i n}$. Both the shape and the magnitude are in agreement with the experimental data shown on Fig 4a [2]. For the lower power level, $\operatorname{Seg}_{0}(\omega)$ is slightly dependent on the $R F$ power $P_{i n}$ and close to the spectral density measured in linear operation. However, there is a strong effect on its magnitude for higher level of $P_{i n}$, especially for frequency deviations where the $G R$ noise components are of primary importance. Moreover, we observe on Fig. 5 that the spectral densities of the up-converted low frequency Generation-Recombination noise sources (around the RF frequency and for $\left.\omega=2 . \pi \cdot 10^{3} \mathrm{rd} / \mathrm{s}\right)$ are reaching values in the same order of magnitude to those of $\operatorname{Seg}_{0}(\omega)$ for the highest level of $P_{i n}$.

\section{CONCLUSION}

Using a microscopic approach, low frequency noise conversion mechanisms in FET have been studied and compared with experimental data for the first time. The results have shown that the spectral density of the base-band voltage noise at the input of the device is strongly dependent of the input power level. In addition, the model is able to describe an important effect : the up-conversion of base-band $G R$ microscopic noise sources located in the channel around the $R F$ frequency at the device electrodes. Note that such approach can be used as well to model accurately phase noise performances of FET's oscillator [3].

\section{REFERENCES}

[1] F. DANNEVILLE, G. DAMBRINE, A. CAPPY, "Noise Modeling in MESFET and HEMT Mixers Using a Uniform Noisy Line Model”, IEEE Trans. on Electron Devices, vol. 45, n. 10, pp. 2207-2212, 1998.

[2] O. LLOPIS , J. VERDIER, R. PLANA, J. GRAFFEUIL ,"Low Frequency Noise in FET devices operated under Nonlinear Conditions ; consequences on oscillator phase noise", Proceedings of the 1995 EuMC, pp. 285-289.

[3] J. OBREGON, M. PRIGENT, J.C. NALLAMTABY, CAMIADE M., RIGAUD D., QUERE R., "Key aspects of modeling and design of low phase noise microwave transistor-oscillators", 2000 (June) IEEE MTT-S International Microwave Symposium, Workshop on Microwave Oscillators, Boston. 


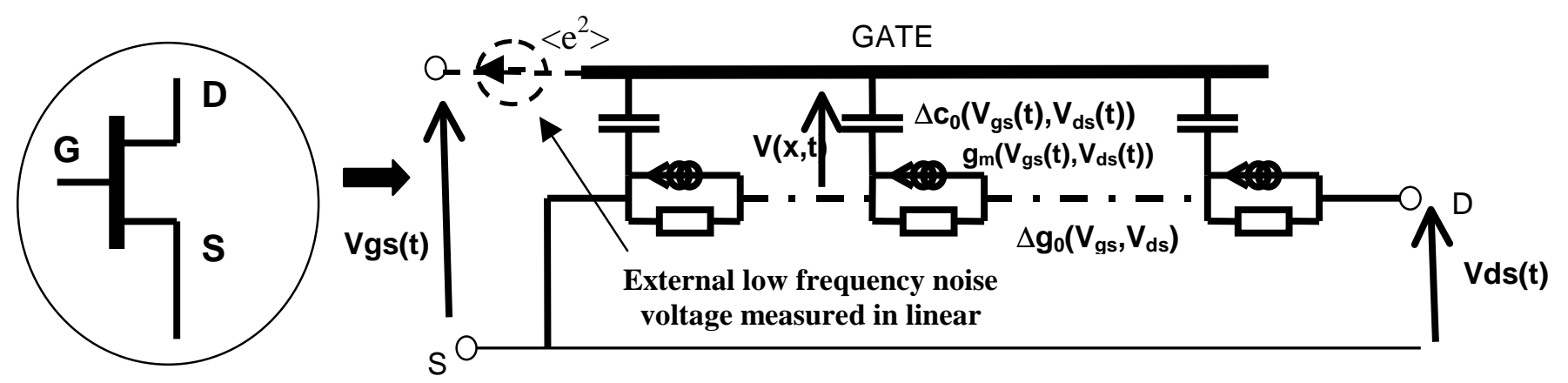

Fig. 1 : FET under large signal operation represented by an active line

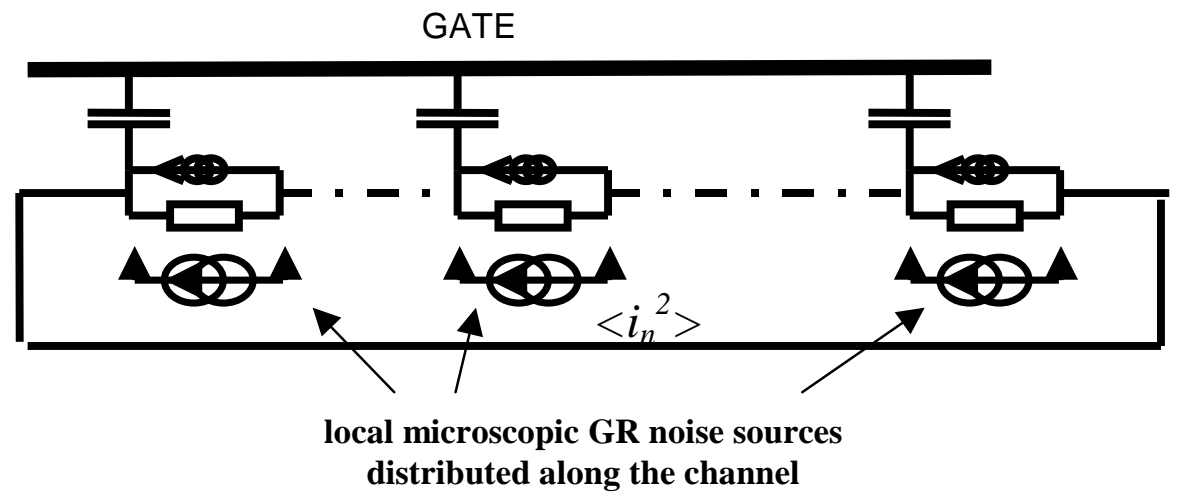

Fig. 2: Circuit used for noise calculation

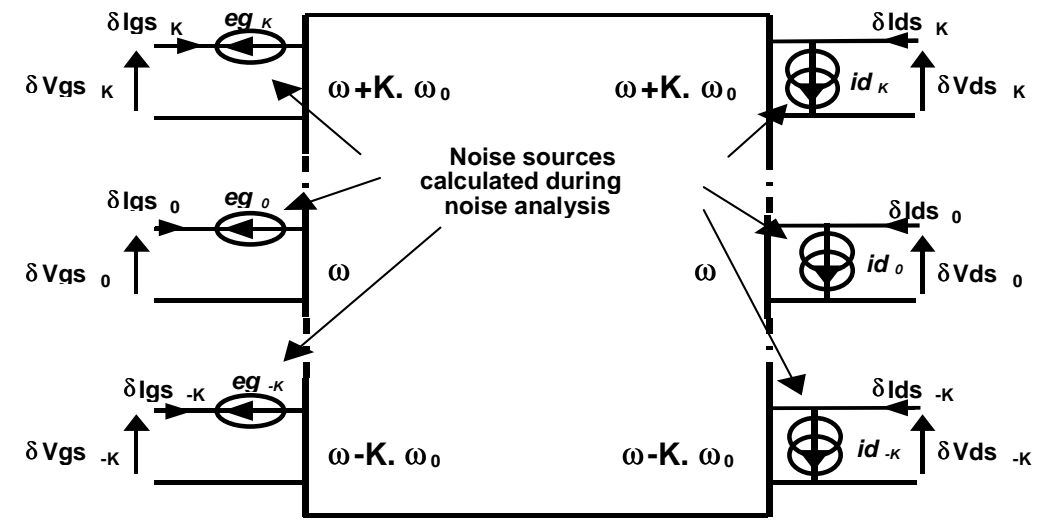

Fig. 3 : FET representated by a linear noisy multifrequency network. 


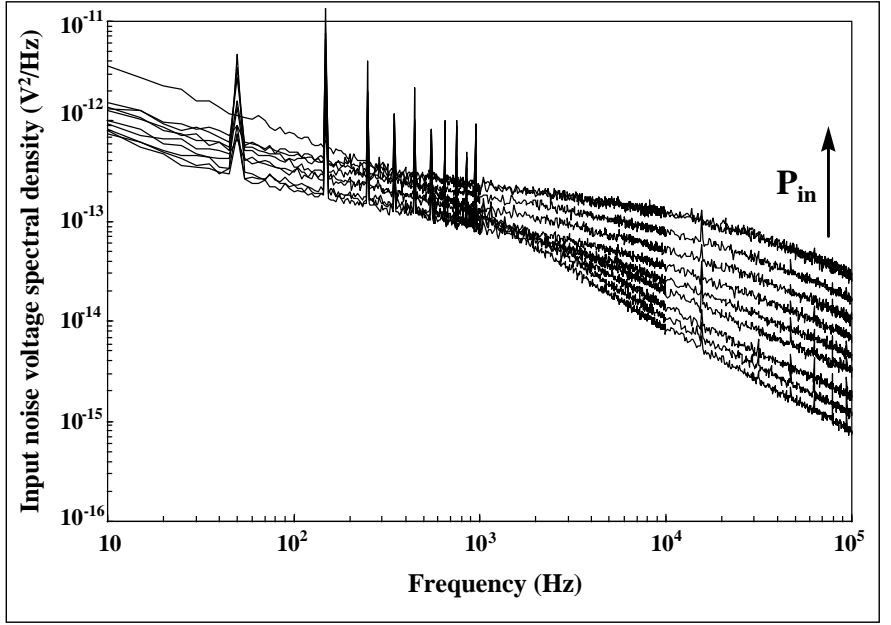

(a)

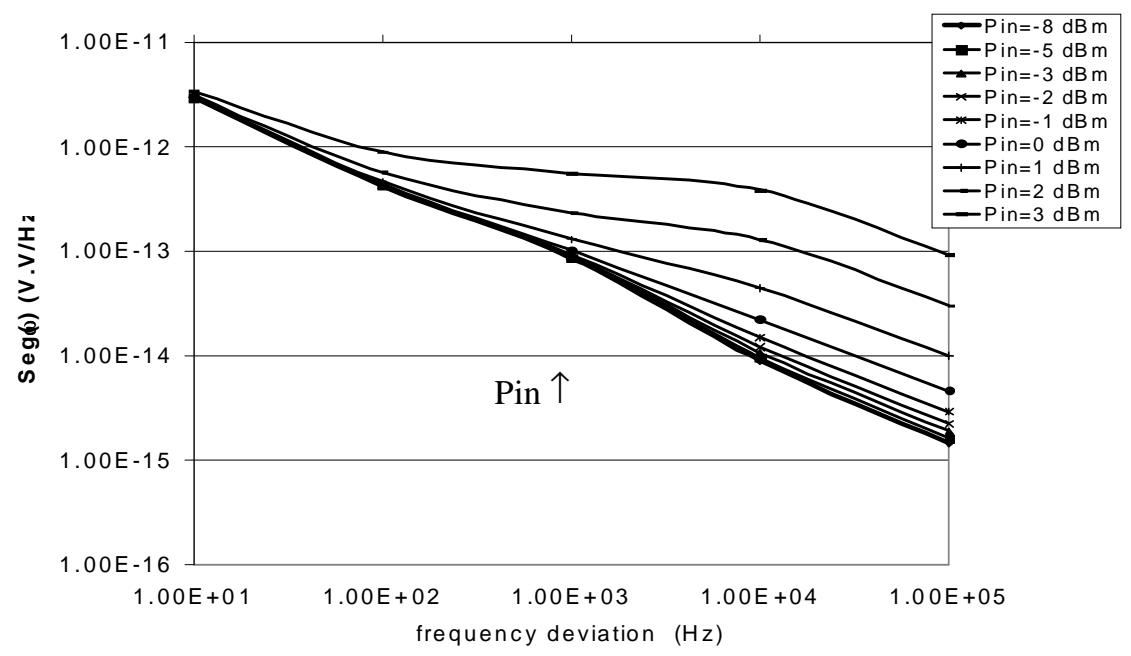

(b)

Fig. 4 : Equivalent input LF noise spectral density measured (a) $\left(-8 \mathrm{dBm}<\mathrm{P}_{\text {in }}<4 \mathrm{dBm}\right)$ and modeled (b) versus different input RF power at $3.5 \mathrm{GHz}$

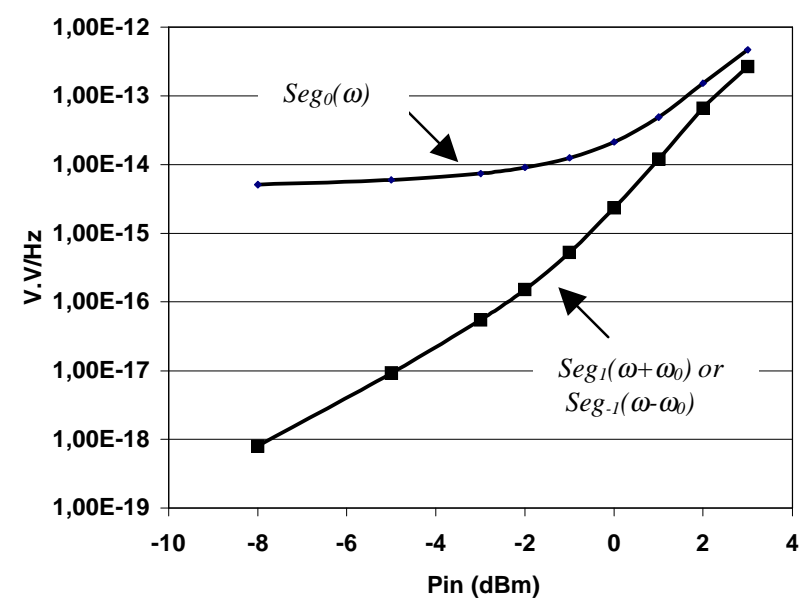

$\underline{\text { Fig. } 5}$ : Base-band $\left(\operatorname{Seg}_{0}(\omega)\right)$ and Up-converted $\left(\operatorname{Seg}_{l}\left(\omega+\omega_{0}\right)\right.$ or Seg-1 $\left.\left(\omega+\omega_{0}\right)\right)$ spectral densities of the input gate noise voltage as a function of the input microwave Power $P_{i n}$. 